\title{
Die Asteriden Sammlung des Museums zu Amsterdam
}

\author{
VON
}

\author{
D . C. PH. SLUITER.
}

Die Asteriden Sammlung, welche jetzt in den Räumen des Museums der Kön. Zoologischen Gesellschaft "Natura Artis Magistra" aufgestellt ist, besteht erstens aus der alten Sammlung der eben genannten Gesellschaft, hauptsächlich tropisch Aziatische und Amerikanische Formen enthaltend, zweitens aus den von Bleeker und später von mir im Indischen Archipel gesammelten Tieren, drittens aus der früheren Universitäts Sammlung, und viertens aus dem an Individuen wenigstens, sehr reichen Material der letzten vier Niederländischen Expeditionen nach dem Nördlichen Eismeer, und zwar nach der Barents See und Kara See.

Obgleich es kaum zu erwarten war, dass sich bei der Bearbeitung dieser Sammlung viel Neues herausstellen würde, so stiess ich doch auf mehrere neue Fundorte, und auch auf einige Formen, welche, so viel ich habe ermitteln können, noch nicht beschrieben wurden, unter welchen besonders ein neuer Solaster aus der Barents See, als Zwischenform zwischen den verschiedenen Genera, welche in der Familie der Solasteridae aufgestellt sind, bemerkenswert ist. Ferner nenne ich noch eine neue sehr eigentümliche Culcita Art und ein von mir zu Pentacer opsis gebrachtes Tier von den Molukken.

Ich werde mich in der nachfolgenden Liste an der Einteilung und Nomenclatur von Sladen ${ }^{x}$ ) halten, wo auch die weitere Synonimie nachzusehen ist.

Im ganzen sind 101 verschiedene Arten vertreten, welche zu 42 Genera gehören. Als neue Formen sind aufgeführt: 2 Astropectens, 1 Pentaceropsis, 1 Culcita und 1 Solaster.

\section{ARCHASTERIDAE.}

1. Pontaster tenuispinus (Düb. u. Kor.) Sladen. Zahlreiche Exemplare in Alkohol aus der Barents See von den verschiedensten Localitäten. Ausserdem noch zahlreiche Exemplare, aber durchweg etwas kleinere, aus der Kara See, $71^{\circ}$ NB. und $63^{\circ}-65^{\circ}$. OL., aus einer Tiefe von 50-90 Faden, von welcher Localität sie bis jetzt noch nicht erwähnt wurden. Unter den mehr als hundert Exemplaren, welche mir unter den Augen kamen, traf ich keine, welche mit der Varietät P. platynota Sladen von den Faröer und Ierland übereinstimmen.

2. Plutonaster (subg. Tethyaster) parelii (Düb. u. Kor.) Sladen. Ein Exemplar in Alkohol von Bergen (Weber).

3. Pseudarchaster tessellatus Sladen, var. arcticus (n. var.) Die Art. Ps. tessellatus wurde bis jetzt nur noch von Sladen beschrieben und zwar von Simons Bai, bei der Kap der Guten Hoffnung. Unter den Asteriden, welche von der Niederländischen Nord-Polar Expe-

1) W. Percy Sladen. Report on the Asteroiden. The voyage of H. M. S. Challenger. Vol. XXX. 
dition mitgebracht sind, finde ich aber 6 Asteriden, welche alle aus der Barents See, $72^{\circ} 14^{\prime} \mathrm{NB}$. und $22^{\circ} 30^{\prime} \mathrm{OL}$. Gr., aus einer Tiefe von 150 Faden herstammen, und dem Ps. tessellatus von der Kap der Guten Hoffnung überaus nahe verwandt sind. Bei dem grössten Exemplar war $R=62 \mathrm{~mm}$., $\mathrm{r}=18 \mathrm{~mm}$. Die Verteilung der Paxillen auf dem Rücken ist genau als bei Ps. tessellatus, nur ist die Reihenstellung auf den Armen öfters recht undeutlich. Die Paxillen selbst sind etwas abweichend, da in der Mitte nur selten ein, gewöhnlich zwei oder drei centrale Höckerchen vorkommen, welche von einem Kranze von 7-11 Dörnchen umgeben sind, ausser noch einigen kleineren. Zahl, Form und Bewaffnung der dorsalen und ventralen Randplatten stimmen genau mit Ps. tessellatus. Dasselbe gilt auch von den Bauchplatten, wenn auch die Grenzen der interradialen Platten zuweilen etwas deutlicher zu unterscheiden sind, da die Granula, welche am Rande der Platten stehen, öfters beträchtlich kleiner sind als die grösseren in der Mitte. Die Ambucralpapillen wie bei der erwähnten Form, 6 in der inneren Reihe, 4 stumpfe papillenförmige in der zweiten Reihe. Ausserhalb dieser noch eine undeutliche dritte Reihe, welche in der Granulation des Bauches übergeht. Auf den Mundplatten kommen vier Reihen von 6 Papillen vor, von welchen aber die inneren kaum grösser sind als die äusseren, wie es beim Typus der Fall ist.

4. Archaster typicus M. u. Tr. Zahlreiche Exemplare getrocknet und in Alkohol von den Molukken, Bawean, Batavia und Mauritius. Einzelne mit vier Armen.

In seiner jüngsten Zusammenstellung der von Pictet und Bedot auf Ambon gesammelten Asteriden, sagt P. de Loriol, dass er unter den zahlreichen Sendungen aus Mauritius immer nur A. angulatus M. u. Tr. gefunden hat und nie A. typicus M. u. Tr., welche ich von dort erwähnte. Ich besitze jetzt noch drei Exemplare von Mauritius, welche von Herrn Robillard gesammelt sind, und welche jedenfalls viel mehr mit A. typicus als mit A. angulatus übereinstimmen. Das Verhältniss des grossen und kleinen Radius ist als 1:5. Es kommen bei einem Exemplar 40 Randplatten vor, bei einem anderen 38. Die Stacheln auf den ventralen Randplatten sind zuweilen doppelt auf einer Platte, grösstenteils aber einfach, zuweilen sind sie vorn breit abgestumpft, zuweilen aber spitz. Die Furchenpapillen verhalten sich genau wie Müller und Troschel es für A. typicus angeben. Die grossen schlanken Pedicellarien sind bei den drei Exemplaren aber sehr regelmässig entwickelt zwischen den beiden Reihen Furchenpapillen, wie das typisch für den A. angulatus von Müller und Troschel ist. Wie aber de Loriol ${ }^{1}$ ) schon hervorhebt sind die Pedicellarien sehr ungleich zahlreich entwickelt. Andrerseits besitze ich Exemplare von A. typicus von den Molukken, welche das Verhältniss der Arme als $1: 6^{1 / 2}$ besitzen, mit 55 dorsalen Randplatten versehen sind, und die Anordnung der Pedicellarien genau so aufweisen wie die Tiere von Mauritius. Alle mögliche Übergänge zwischen den beiden Formen sind zu finden, und ich glaube nicht, dass beide als gesonderte Arten beibehalten werden können. Auch Sladen ${ }^{2}$ ) erwähnt schon einige Übergänge zwischen beiden Formen.

\section{PORCELLANASTRIDAE.}

5. Ctenodiscus corniculatus (Linck) Perrier. Mehrere Exemplare aus der Barents See, $70^{\circ} 17^{\prime}$ NB., $46^{\circ} 31^{\prime}$ OL. Gr. und zahlreiche Exemplare aus der Kara See, $71^{\circ} \mathrm{NB}$., 63' OL. aus einer Tiefe von 45-57 Faden. Alle in Alcohol, und drei von Bodö, Norwegen (Weber).

\section{ASTROPECTINIDAE.}

6. Craspidaster hesperus (M. u. Tr.) Sladen. Zahlreiche Exemplare in Alcohol von der

1) P. de Loriol. Catalogue raissoné des Echin. recueillis à l'Ile Maurice. II. Genève 1885, pg. 79.

2) P. Sladen. l. c. 
Bai "von Batavia ans einer Tiefe von 5-14 Faden (Sluiter). Nach der jetzt vorliegenden ausführlichen Beschreibung und Abbildung dieser Art von Sladen, bin ich zu der Überzeugung gekommen, dass die früher von mir als Astropecten macer beschriebene Form mit dem alten Archaster hesperus M. Tr. identisch ist. Die Zahl und Form der interradialen Bauchplatten kann aber mehr oder weniger verschieden sein, und bei grösseren Exemplaren, bis 4 mehr betragen als von Sladen beschrieben und abgebildet wird.

7. Leptoptychaster arcticus (Sars) Sladen. Sehr zahlreiche Exemplare in Alkohol von der Barents See, $72^{\circ} 14^{\prime}-72^{\circ} 36^{\prime}$ NB. $22^{\circ} 30^{\prime}-25^{\circ} 58^{\prime}$ OL. Gr. aus einer Tiefe von 140-155 Faden.

8. Astropecten irregularis Linck. Mehrere Exemplare in Alkohol vom Doggersbank und von der Nord See.

9. Astropecten aurantiacus (Linné) Gray. Ein Exemplar von Rovigno in Alkohol, und zwei getrocknet ohne Angabe von Herkunft.

10. Astropecten polyacanthus M. u. Tr. Ein Exemplar von dem Roten Meer und zwei von den Molukken (v. d. Hucht), alle getrocknet.

11. Astropecten alatus. Perrier. Ein Exemplar in Alkohol von Ambon (Bleeker).

12. Astropecten javanicus Lütken. Drei Exemplare in Alkohol von der Bai von Batavia (Sluiter).

13. Astropecten ternatensus (n. sp.). Zwei Exemplare getrocknet von den Molukken (v. d. Hucht). Fünf Arme, $R=50 \mathrm{~mm} . \mathrm{r}=16 \mathrm{~mm}$. Die Arme an der Basis $17 \mathrm{~mm}$. breit, nach der Spitze zu sich nur sehr langsam verjüngend. Die Spitze selbst bleibt stumpf. Die Armwinkel nicht ausgerundet. Das Paxillenfeld des Rückens bleibt auch auf den Armen breit, so dass es auf der Hälfte der Armlänge noch $8 \mathrm{~mm}$. misst, da die dorsalen Randplatten nur wenig auf die Rückenfläche übergreifen. Die Paxillen auf der Mitte der Scheibe, wie gewöhnlich kleiner als die am Rande. Jede Paxille hat ein centrales Höckerchen und davon ausstrahlend 6 oder 7 sehr lange Dörnchen.

Die 26 dorsalen Randplatten sind grob gekörnt, und jede derselben trägt zwischen den Granula noch zwei oder drei deutlich hervorragende Dörnchen. Die 27 ventralen Randplatten sind dicht mit platten schuppenförmigen Stachelchen besetzt. Aủs dieser Bewaffnung ragt auf jeder Platte eine Reihe grösserer, platter Stacheln hervor, welche nach dem Rande zu allmählig die Länge erreichen der ziemlich grossen Randstacheln. Diese letzteren sind in den Armwinkeln deutlich abgeplattet und lanzetförmig, nach der Mitte der Arme zu. werden sie aber schon mehr conisch.

Die Bewaffnung der Ambulacralfurchen besteht aus einer inneren Reihe von 3 langen schlanken Papillen, von welchen die mittlere etwas länger ist als die beiden seitlichen. Dann folgen auf jeder Ambularcalplatte nach aussen zu fünf oder sechs ungleich lange Papillen, welche aber keine Reihe bilden, sondern mehr in einem Haufen stehen. Diese Papillen sind deutlich spatelförmig, mit einem dünnen Stiel auf die Platte eingepflanzt und in eine platte ziemlich breite Schuppe endigend. Auch die Dörnchen auf den ventralen Randplatten haben in der Nähe der Furchen deutlich diese Spatelform, sind aber viel kleiner als die auf den Ambulacralplatten selbst.

An der Basis der Arme hat sich zwischen den ventralen Randplatten und den Ambulacralplatten jederseits eine einfache Reihe von etwa 12 kleinen ventralen Platten eingeschoben, welche ebenso mit kleinen spatelförmigen Dörnchen bedeckt sind. Die Bewaffnung der Mundplatten war an den getrockneten Exemplaren nicht genau mehr zu ermitteln, bestand aber jedenfalls aus vier Reihen von mehreren abgeplatteten Dörnchen. 
Die Farbe der beiden getrockneten Lxemplaren war auf dem Rücken graubraun. An den ventralen Randplatten war auf mehreren Stellen ein helleres Orangebraun bewahrt geblieben.

Der allgemeine Habitus, die Form und Bewaffnung der dorsalen und vențralen Randplatten stimmt fast genau überein mit der gewöhnlichen A. irregularis unserer Europäischen Meere, und auf den ersten Blick könnte sie fast damit verwechselt werden. Die Form der Rückenpaxillen so wie die eigentümliche Bewaffnung der Ambulacralplatten ist aber eine recht verschiedene.

14. Astropecten ornans (n.sp.). Zwei getrocknete Exemplare, ein von Neu-Ierland und ein von Neu-Caledoniën. Fünf Arme, $R=38 \mathrm{~mm}, \mathbf{r}=11 \mathrm{~mm}$. Scheibe ziemlich gross, Arme an der Basis breit, $12 \mathrm{~mm}$, am ersten $\% / \mathrm{s}$ Teil sich nur wenig verjüngend, dann schneller an Breite abnehmend, und spitz endigend. Die Form der Arme erscheint dadurch schwach lanzetförmig. Die Armwinkel schwach ausgerundet.

Die Paxillen des Rückens stehen auf der Scheibe ganz regellos, auf den Armen, wenigstens an den Seiten derselben, in regelmässigen Querreihen. Jede Paxille trägt in der Mitte 1-3 centrale Höckerchen, einzelne sogar vier. Am Rande stehen 8-14 ziemlich lange Dörnchen.

Die 34 dorsalen Randplatten sind mit gleichmässigen Granula bedeckt, ohne jegliche weitere Bewaffnung. Nur die Granula am Rande jeder Platte sind etwas schlanker und länger als die mittleren. Die Platten selbst sind höher als breit, nur an den Armspitzen mehr viereckig. In den Armwinkeln liegen sie fast ausschliesslich auf der Seitenwand, nach der Mitte der Arme zu, greifen sie aber mehr und mehr auf die Rückenseite über, und liegen bei den stark abgeplatteten Armspitzen fast ganz auf dem Rücken.

Die ventralen Randplatten sind gleich zahlreich als die dorsalen und denselben regelmässig gegenübergestellt. Sie ragen aber über den Verwachsungsrand mit den dorsalen Randplatten ziemlich weit hervor zur Bildung einer Franze, wie es etwa auch bei Astropecten Richardi Perrier vorkommt. Diese hervorragende Franze ist auf der Rückenseite mit gleichen Granula bedeckt als die dorsalen Randplatten. An der ventralen Seite ist jede Platte umsäumt von einer Reihe sehr feiner Dörnchen, und ausserdem kommen auf dem der Armspitze zugekehrten Rande noch 5-7 grössere spitze conische Stachelchen vor. An dem freien seitlichen Rande liegen zuerst 3-5 kleine spitze Stachelchen und dann am äusseren Rande die eigentlichen Randstacheln, von welchen immer zwei auf jeder Platte stehen. Diese Randstacheln sind abgeplattet und an ihr Ende abgestumpft. Die an der Basis der Arme sind die grössten, wodurch die Lanzetform der Arme noch deutlich wird.

Auf jeder Ámbulacralplatte steht zuerst eine innere Reihe von drei sehr dünnen Papillen, von welchen die mittlere etwa doppelt so lang ist als die beiden anderen. Etwas weiter von der Ambulacralfurche entfernt steht auf jeder Platte ein kräftiger Stachel, und um diesen noch einige viel dünneren und kürzeren.

Die Bewaffnung der Mundplatten besteht aus vier Reihen von Papillen. Am Rande jeder Platte liegt jederseits eine Reihe von etwa zehn Papillen, und auf der Mitte der Platte noch eine doppelte Reihe mit viel selteneren. Am inneren Mundwinkel liegen 3 beträchtlich grössere Papillen, von welchen wieder die mittlere die grösste ist.

Die Madreporenplatte ist ziemlich gross, mit von der Mitte ausstrahlenden Furchen versehen, dem Rande der Scheibe nahe liegend, so dass nur eine oder zwei Paxillenreihen zwischen ihr und die dorsalen Randplatten kommen.

Wie aus obiger Beschreibung hervorgeht steht diese Form jedenfalls in der Nähe der drei von Perrier beschriebenen Astropecten Arten: A. Richardi; A. alatus und A. spatuliger. Am meisten gleicht sie den A. Richardi von Cayenne, weicht aber auch von dieser Form noch beträchtlich $a b$ in Betreff der Form der Rückenpaxillen, der Bewaffnung der ventralen Randplatten so wie des Verhältnisses des Armradius zum Scheiberadius.

15. Luidia columbiae (Gray) Perrier. Ein Exemplar von Californien in Alkohol. 
16. Luidia hardwickii (Gray) Perrier. Ein Exemplar aus der Bai von Batavia in Alkohol (Sluiter).

17. Luidia maculata M. u. Tr. Ein Exemplar aus der Bai von Batavia in Alkohol (Sluiter).

\section{PENTAgONASTERIdAE.}

18. Pentagonaster astrologorum (M. Tr.) Perrier. Drei getrocknete Exemplare von Australiën.

19. Pentagonaster granularis (Retzius) Perrier. Zahlreiche Exemplare von der Barents See, $72^{\circ} 36^{\prime}$ NB., $24^{\circ} 54^{\prime}$ OL. und mehreren anderen in der Nähe liegenden Stellen, Tiefe 140 Faden, 12 Exemplare von Norwegen (M. Weber), und 3 getrocknete Exemplare aus dem Nördlichen Eismeer.

20. Nectria ocellifera (Lam.) Gray. Ein erwachsenes Exemplar in Alkohol von Ambon (Bleeker). Bỉs jetzt war die Gattung Nectria nur noch erwähnt von Bass-Strasse, Tasmania, Fiji Inseln und von Port Philip. Das Vorkommen dieser merkwürdigen Form auch im Indischen Archipel ist also nicht ohne Interesse. Das Ambonsche Exemplar stimmt, in Betreff der allgemeinen Körperform, $R=2^{1 / 2} r$, mit $N$. ocellifera überein, aber in Betreff der Granulation der Rückentuberkel, des Netzes der Rückenplatten, der Form und Lage der Madreporenplatte, und der Ambulacralpapillen mehr mit N. ocellata (Perrier). Auch mir scheint die Vermutung Sladen's, dass die von Perrier aufgestellte Art N. ocella ta specifisch nicht von N. ocellifera zu trennen ist, sehr wahrscheinlich.

21. Stellaster incei Gray. Drei Exemplare, und zwar ein in Alkohol von Java (Bleeker), ein in Alkohol aus der Bai von Batavia (Sluiter), und ein getrocknet von Japan (Frank).

22. Goniodiscus Sebae M. u. Tr. Sechs Exemplare, und zwar drei in Alkohol von Ambon (Bleeker), und drei getrocknet von den Molukken (v. d. Hucht).

\section{ANTHENEIDAE.}

23. Anthenea pentagonula (Lam.) Perrier. Ein getrocknetes Exemplar von China.

24. Anthenea flavescens (Gray) Perrier. Ein getrocknetes Exemplar von den Molukken (v. d. Hucht).

25. Hippasteria plana (Linck) Gray. Zwei getrocknete Exemplare von der Küste Norwegens, ein junges Exemplar in Alkohol von Bergens Fjord (M. Weber), und sieben Exemplare in Alkohol aus der Barents See $72^{\circ} 29^{\prime}$ NB., $24^{\circ} 57^{\prime}-25^{\circ} 58^{\prime}$ OL., Tiefe 140 Faden.

\section{PENTACEROTIDAE.}

26. Pentaceros affinis M. Tr. Ein Exemplar, getrocknet von den Molukken (v. d. Hucht).

27. Pentaceros grayi Bell. Zwei Exemplare, getrocknet von den Molukken (v. d. Hucht), vier in Alkohol von Ambon (Bleeker) und ein in Alkohol von Billiton (Sluiter).

28. Pentaceros hiulcus M. u. Tr. Vier getrocknete Exemplare und zwei in Alkohol von 
den Molukken (v. d. Hucht), 26 Exemplare in Alkohol von den Molukken (Bleeker), ein Exem. plar in Alkohol von Billiton (Sluiter).

29. Pentaceros muricatus Linck. Ein getrocknetes Exemplar von Neu-Ierland, zwei getrocknete von den Molukken. (v. d. Hucht), und ein in Alkohol von Zanzibar.

30. Pentaceros productus Bell. Ein Exemplar getrocknet von Ost-Indien ohne genauere Angabe. Das Exemplar dieser merkwürdigen Art aus dem hiesigen Museum ist noch etwas grösser als die von Bell beschriebenen Tiere von Billiton, da $R=250 \mathrm{~mm}$. und $\mathrm{r}=56 \mathrm{~mm}$. war. Übrigens stimmt das Exemplar genau mit Bell's Beschreibung, nur haben die Papillen in der Mittellinie an der Basis der Arme nackte Zipfel, wie die von Sladen erwähnte Varietät P. tuberata von den Philippinen.

\section{Pentaceros reinhardti Lütk. Ein Exemplar in Alkohol von Ternate (Bleeker).}

32. Pentaceros reticulatus Linck. Neun Exemplare getrocknet von St. Domingo. Bei drei sehr grossen Exemplaren war durch zusammendrücken die Form der Tiere zu einer flachen fünfeckigen Scheibe verunstaltet, wie es auch von Bell für die als P. lapidarius Grube hervorgehoben wịd, welche Form auch nicht anders als die gewöhnliche P. reticulatus ist.

33. Pentaceros sladeni de Loriol. Ein Exemplar getrocknet von den Molukken (v. d. Hucht). Bis jetzt war diese Art nur noch von Mauritius bekannt.

34. Pentaceros tuberculatus M. u. Tr. Ein Exemplar in Alkohol von Zanzibar.

35. Pentaceros turritus Linck. Vier Exemplare getrocknet von den Molukken und acht in Alkohol von derselben Inselgruppe (Bleeker).

36. Pentaceropsis euphues n. sp. Ein getrocknetes, wahrscheinlich noch junges Exem. plar von den Molukken (v. d. Hucht).

Fünf Arme. $R=26 \mathrm{~mm}$., $\mathrm{r}=5 \mathrm{~mm}$. Arme lang und schlank, an der Basis $6 \mathrm{~mm}$. breit, sich allmählig nach der Spitze verjüngend. Der Rücken wenig erhaben. Die Rückenplatten abgerundet mit feiner gleichmässiger Granulation, auf der Scheibe unregelmässig gelagert, auf der Basis der Arme in dreidoppelter Reihe, am letzten Viertel aber wieder unregelmässig. Auf der Mitte der Scheibe stehen vier grössere Tuberkel dicht neben einander, den After zwischen sich fassend. Auf der Mittellinie der Arme stehen auf dem ersten $2 / 5$ Teil drei grössere Tuberkel, eine Reihe bildend. Nach der Armspitze zu kommen dann noch fünf oder sechs unregelmässig stehende hinzu. Am ersten 2/5 Teil wechselt regelmässig eine tuberkeltragende mit einer unbewaffneten Platte ab. Die Tuberkel sind an der Basis fein granulirt, aus welcher Granulation eine nackte Spitze hervorragt. Zwischen den Platten liegen die unregelmässig dreieckigen kleinen Porenfelder mit nur wenigen, 2-4, Poren.

Die Bauchplatten sind mit polygoner Granulation bedeckt. Die auf der Mitte der Platte stehenden Granula sind beträchtlich grösser als die am Rande, wodurch die verschiedenen Bauchplatten deutlich zu unterscheiden sind, wenn auch ohne scharfe Grenze. Bis auf etwa die Hälfte des Armes schiebt sich eine Reihe kleiner Bauchplatten zwischen den ventralen Randplatten und den Ambulacralplatten ein.

Die 15 dorsalen Randplatten sind den Rückenplatten gleich granulirt und abwechselnd mit einem grossen Tuberkel versehen und unbewaffnet. Die unbewaffneten Platten sind viel kleiner als die bewaffneten. Die letzten drei an der Armspitze sind aber alle unbewaffnet, so dass sechs grössere Tuberkel auf jeder Armkante kommen. Die 16 ventralen Randplatten sind mit gleicher Granulation als die dorsalen bedeckt. Fast alle tragen Tnberkel mit nackter Spitze, welche aber 
viel kleiner sind als die Tuberkel der dorsalen Randplatten. Nur die zwei in den Armwinkeln sind unbewaffnet. Zwischen dorsalen und ventralen Randplatten liegt in den Armwinkeln und bis zur Hälfte des Armes zu verfolgen, jederseits eine Reihe von etwa 9 unbewaffneten kleinen Platten mit der gleichen Granulation als die Rand- und Rückenplatten bedeckt.

Die Ambulacralplatten tragen eine innere Reihe von 4 längeren etwas kolbig angeschwollenen Papillen, und eine äussere Reihe von 2 oder 3 dickeren aber kürzeren. Eine dritte Reihe ist kaum zu unterscheiden. Die Bewaffnung der Mundplatten ist die gewöhnliche für die Pentacerotiden.

Zwei Madreporenplatten mit unregelmässig verlaufenden Furchen, auf zwei auf einander folgenden interbrachialen Winkeln, etwa auf der Mitte vom Centrum zum Armwinkel.

Es ist allerdings nur mit einem gewissen Zweifel, dass ich das oben beschriebene 'Tier zu der Gattung Pentaceropsis bringe, welche von Sladen zur Aufnahme der abweichenden Form, Oreaster obtusatus M. u. Tr. aufgestellt wurde. Es ist hauptsächlich die Reihe Platten, welche zwischen ventrale und dorsale Randplatten eingeschoben ist, was mich dazu bringt. Auch die Anordnung der Rückenplatten kommt einigermassen überein mit dem zweiten Typus von Pentaceros obtusatus, wie Perrier diesen beschrieben hat. In dem Verhalten der Randplatten und namentlich durch den Besitz von verhältnissmässig grossen Tuberkeln, weicht diese Form aber beträchtlich von dem P. obtusatus ab. Wahrscheinlich ist aber das beschriebene Exemplar noch jung, und könnte sich einiges bei den erwachsenen Tieren etwas ändern.

37. Nidorellia michelini Perrier. Ein Exemplar, getrocknet von Panama.

38. Culcita çoriacea M. u. Tr. Ein Exemplar, getrocknet vom roten Meere.

39. Culcita novae guineae M. u. Tr. Zwei getrocknete Exemplare von den Molukken (v. d. Hucht), neun in Alkohol von den Molukken (Bleeker) und zwei in Alkohol von der Bai von Batavia (Sluiter). Es ist nur mit einem gewissen Zweifel, dass ich alle diese Exemplare zu derselben Species C. novae guinea e rechne. Die sorgfältige und ausführliche Besprechung der $\mathrm{Culcita-Arten}$ von Hartlaub ${ }^{1}$ ) zeigt aufs deutlichste, wie ${ }^{-s c h w e r}$ es ist die verschiedenen $\mathrm{Cul-}$ cita-Species scharf aus einander zu halten. Unter den dreizehn Exemplaren, welche ich vor mir habe, besitzen sechs die typische grobe nicht gruppenständige Granulation der Bauchseite. Die Porenfelder des Rückens sind rosettenständig, gross und mit einer Neigung zur Verschmelzung. Die Rückentuberkel zwischen den Porenfeldern sind grösser als die auf den Porenfeldern. Bei drei weiteren Exemplaren von den Molukken ist die Granulation des Bauches etwa die gleiche, nur sind die Granula noch etwas grösser. Die Porenfelder des Rückens sind aber überall durch ziemlich breite Zwischenräume scharf von einander getrennt. Diese Zwischenräume sind sehr fein granulirt, während aus der feinen Granulation mehrere vereinzelt stehende grosse Tuberkel hervorragen. Ein von mir in der Bai von Batavia gesammeltes Exemplar stimmt mit diesen drei zuletzt erwähnten überein, nur dass die grösseren Tuberkel auf dem Rücken gänzlich fehlen. Die ganze Rückenseite gleicht also genau der photografischen Abbildung, welche Hartlaub von C. grex giebt, und wie ich dieselbe auch mit dem Leidener Original Exemplar habe vergleichen können. Ich möchte denn auch das Tier zur C. grex rechnen, wenn es nicht durch die grobe Granulation des Bauches und die hohe gewölbte Form doch wieder der C. novae guineae näher stand. Ein zweites Exemplar aus der Bai von Batavia zeigt wieder, in Betreff der Verteilung der Porenfelder auf dem Rücken, ein Zwischenstadium zwischen diesen beiden Extremen. Bei einem jüngeren Exemplar von den Molukken (Bleeker) stehen die Granula des Bauches deutlich in Gruppen, während die Porenfelder und Tuberkel des Rückens sich verhielten wie bei den sechs zuerst erwähnten Tieren von den Molukken.

Indem ich die Tiere längere Zeit lebend beobachtet habe, ist es mir nicht möglich, der allgemeinen Körpergestalt den Wert beizulegen, wie Hartlaub es thut. Dasselbe Tier kann seine

1) C. Hartlaub, Ueber die Arten und den Skelettbau von Culcita. Notes from the Leiden Museum. Vol. XIV fig. 65. 
Gestalt derartig ändern, dass es zuweilen hochgewölbt, zuweilen flach scheibenförmig erscheinen kann. Kränkliche Tiere sind immer scheibenförmig, wodurch auch alle Exemplare, welche nicht unmittelbar, nachdem sie gefangen sind, in Alkohol versetzt wurden, scheibenförmig erscheinen, was bei vielen Museumstücken wohl der Fall gewesen sein,wird.

Die früher von mir ${ }^{1}$ ) als C. schmideliana erwähnten Tiere aus der Bai von Batavia, sind, wenigstens der geografischen Verbreitung nach, auch wohl zu C. novae guinea zu zählen. $\mathrm{Ob}$ aber die beiden Arten, wenn das Vergleich-Material sich mehrt, als gesonderte Arten aus einander zu halten sind, scheint mir sehr zweifelhaft. Von de Loriol (l.c. p. 182) wurde ein von Pictet und Bedot gesammeltes Exemplar als C. grex bestimmt, aber noch keine Rücksicht genommen von der ausführlichen Arbeit von Hartlaub.

40. C. niassensis n. sp. Ein getrocknetes Exemplar von der Insel Nias. Obgleich die ursprüngliche Form offenbar durch das Trocknen sehr gelitten hat, ist doch das für eine $\mathrm{Cul-}$ cita sehr stark Vorspringen der fünf Arme schwerlich ausschliesslich darauf zurück zu führen. Bei dem trocknen Exemplar ist $R=90 \mathrm{~mm}$., $r=50 \mathrm{~mm}$., ein Verhalten, wie es noch bei keiner Culcita gefunden wurde. Der Rücken scheint mit einer gleichmässigen feinen Granulation bedeckt gewesen zu sein, welche aber bei dem. einzigen vorliegenden Exemplar grösstenteils abgerieben ist, so dass jetzt der Rücken fast glatt erscheint.

Diese nackte Haut erscheint jetzt tief schwarz, was aber vielleicht nicht die ursprüngliche Farbe darstellt. Die Poren sind auf der jetzt trocknen glatten Rückenhaut nur sehr schwer zu unterscheiden. Es scheint mir aber, dass sie auf dem Rücken in Felder gestanden haben. An den Seiten des Körpers, wo die Granulation besser bewahrt geblieben ist, stehen die Poren aber mehr regelmässig verteilt, ohne Porenfelder zu bilden. Grössere Tuberkel kamen auf dem Rücken wohl gar nicht vor. Auch die Bauchfläche ist mit einer sehr gleichmässigen Granulation bedeckt. Die Granula sind deutlich polygon und schliessen dicht an einander, wodurch ein sehr fein gezeichnetes Plaster entsteht. Die Granula bei den Mundwinkeln und den Ambulacren sind die grössten. Nach dem Rande zu werden sie allmählig feiner und gehen in die sehr feine Granulation der Seiten und des Rückens über.

Sehr eigentümlich ist die Einrichtung der Pedicellarien. Jede Pedicellarie besteht nämlich aus einer fast kreisrunden Alveole mit einer ovalen Öffnung, welche an den Kalkplatten des Skeletts fest gewachsen sind. In diesen Alveolen liegen die zwei breiten am Rande gezähnten Klappen, welche sich, wie es scheint gänzlich in den Alveolen zurückziehen können. Die Klappen scheinen sich bei den trocknen Tieren leicht abzulösen, da bei den meisten Alveolen dieselben verschwunden waren. Es häufen sich diese Pedicellarien hauptsächlich bei den Mundwinkeln, und auf die erste Hälfte der Arme, den Ambulacralfurchen entlang. Ausserdem kommen auf der Bauchfläche und vereinzelt auch auf dem Rücken, noch mehrere kleine gewöhnlich geformte Klappenpedicellarien vor ohne Alveolen. Bekanntlich kommen derartige mit Alveolen versehenen Pedicellarien noch vor bei Ophidiaster pusillus, wo aber die Alveolen eine 8-förmige Gestalt haben und die Klappen einen schmalen Stiel besitzen.

Die Bewaffnung der Ambulacralplatten besteht aus einer inneren Reihe von 7 Papillen, von welchen, von der Armspitze abgerechnet, immer die zweite und dritte die längsten sind, und die folgenden nach dem Munde zu immer kleiner werden, so dass die siebente ziemlich klein ist. Zuweilen kommt noch eine sehr kleine achte hinzu. In der äusseren Reihe stehen fünf Papille auf jeder Platte, von welchen die mittleren die grössten sind. Wie gewöhnlich liegt zwischen zwei Platten eine Pedicellarie; aber diese ist wie die übrigen Pedicellarien der Bauchfläche in einer Alveole eingesenkt, welche in der dem Munde zugekehrten Seite der Ambulacralplatte liegt.

Die Madreporenplatte is ziemlich gross, etwa dreieckig, dem Centrum etwas näher liegend als

1) C. Ph. Sluiter, Die Evertebraten aus der Sammlung in Batavia. Natuurk. Tijdschr. v. Nederl. Indië, Band 48, pg. 305. 
dem Rande, mit unregelmässig radiär verlaufenden Furchen. Die Oberfläche war aber zum Teil zerbrochen.

Das Museum besitzt von dieser merkwürdigen $\mathrm{Culcita-Art} \mathrm{leider} \mathrm{nur} \mathrm{ein} \mathrm{einziges} \mathrm{Exemplar,}$ welches dazu bei der Conservirung oder nachher sehr gelitten hat. Die eigentümliche fünfstrahlige Gestalt, die in Alveolen liegenden Pedicellarien und die polygonalen fein plasterartige Granulation des Bauches sind wohl die am meisten abweichenden Eigentümlichkeiten dieser Art. Der Fundort, die Insel Nias, eine der grösseren Insel westlich von Sumatra, liegt so sehr ausserhalb der gewöhnlichen Reiserouten, dass man befürchten muss, ob in nächster Zeit von dort frisches Material gesammelt werden wird, wesshalb ich mich entschloss die obige Beschreibung des allerdings ungenügend conservirten einzigen Exemplars zu geben.

\section{GYMNASTERIIDAE.}

41. Gymnasterias carinifera (Lam.) v. Martens. Drei getrocknete Exemplare (zwei jüngere und ein erwachsenes) von den Molukken (v. d. Hucht), ein junges Exemplar in Alkohol von Ambon (Bleeker) und ein erwachsenes Exemplar in Alkohol von der Bai von Batavia (Sluiter).

42. Porania pulvillus (O. F. Müller) Norman. Zwei getrocknete Exemplare von der Küste Norwegens.

43. Rhegaster tumidus var. tuberculata (Dan. u. Kor.) Sladen. Fünfzehn Exemplare in Alkohol aus der Barents See von $70^{\circ} 17^{\prime}-76^{\circ} 51^{\prime} \mathrm{NB}$. und $44^{\circ} 21^{\prime}-46^{\circ} 31^{\prime} \mathrm{OL}$. Gr., Tiefe $45-150$ Faden.

44. Poraniomorpha rosea Dan. u. Kor. Vier Exemplare aus der Kara See, $71^{\circ}$ N. B., $64^{\circ}$ OL. Gr. und sechs aus der Barents See, $72^{\circ} 36^{\prime}$ NB., $24^{\circ} 57^{\prime}$ OL. Gr., Tiefe 140 Faden, alle in Alkohol.

\section{ASTERINIDAE.}

45. Asterina cephea (M. u. Fr.) v. Martens. Drei Exemplare, getrocknet, von den Molukken (v. d. Hucht) sechs in Alkohol von Ambon, neun erwachsene und drei junge, alle in Alkohol von der Bai von Batavia (Sluiter).

46. Asterina calcar (Lam.) Gray. Vier Exemplare getrocknet von Neu Ierland (Cumming), ein in Alkohol von Sydney.

47. Asterina gibbosa (Pennant) Forbes. Drei getrocknete Exemplare vom Nördlichen Eismeer (Israel), Grönland, und ein in Alkohol von St. Vaast (Selenka, Reichs Museum) und mehrere von Neapel. Bis jetzt war diese Art nur von den Europäischen und Nord-Afrikanischen Küsten des Atlantischen Ocean und vom Mittelmeer bekannt, mit dem nördlichsten Breitegrade von $60^{\circ}$ N. B.

48. Asterina exigua (Lam.) Perrier. Drei getrocknete Exemplare von den Molukken (v. d. Hucht), ein getrocknetes Exemplar von Australiën (Cumming), ein in Alkohol von der Bai von Batavia (Sluiter), und fünf von unbekannter Herkunft.

49. Asterina marginata (Val.) Perrier. Zwei Exemplare in Alkohol von Brazilien und ein in Alkohol, Fundort unbekannt (Reichs Museum).

50. Asterina regularis Verrill. Ein getrocknetes Exemplar, und zwei Exemplare in Alkohol, alle von Australiën. 
51. Asterina coronata v. Martens. Ein Exemplar in Alkohol von Halmaheira (Bernstein, Reichs Museum):

52. Asterina pectinifera M. Tr. Ein Exemplar in Alkohol von Japan (v. Siebold, Reichs Museum).

53. Palmipes membranaceus Linck. Zwei getrocknete Exemplare von den Europäischen Meeren, ohne genaure Angabe des Fundorts (Damon), und zwei in Alkohol vom Mittelmeer.

\section{LINCKIIDAE.}

54. Fromia milleporella (Lam.) Gray. Drei Exemplare in Alkohol von den Molukken (v. d. Hucht), fünf getrocknet, Fundort unbekannt (Frank) und zwei in Alkohol von der Bai von Batavia (Sluiter).

55. Ophidiaster cylindricus M. u. Tr. Zwei Exemplare in Alkohol von Ambon (Bleeker).

56. Ophidiaster ophidianus Ag. Ein getrocknetes Exemplar von der Insel Madeira (Frank).

57. Ophidiaster purpureus Perrier. Vier Exemplare von den Molukken (v. d. Hucht) und zwei von Neu-lerland (Frank), alle getrocknet.

58. Ophidiaster pusillus M. u. Tr. Zwei getrocknete Exemplare von den Molukken (v. d. Hucht), sechs in Alkohol von den Molukken (Bleeker) und ein in Alkohol von der Bai von Batavia (Sluiter). Die eigentümlichen von Perrier genau beschriebenen Pedicellarien kommen nicht immer in gleich grosser Anzahl vor. Bei dem von mir in der Bai von Batavia gefischten Exemplar waren sie z. B. sehr selten, so dass im ganzen auf einem Arm nur zwei oder drei vorkamen. Aber auch bei den anderen war die Häufigkeit eine recht verschiedene.

59. Leiaster leachii Gray. Ein Exemplar in Alkohol von den Molukken (v. d. Hucht).

60. Linckia ehrenbergi i M. u. Tr. Fünf Exemplare in Alkohol von Mauritius (Robillard), unter welchen eine sehr auffallende Kometenform.

61. Linckia multifora (Lam.) v. Martens. Vier getrocknete Exemplare von Flores (Museum Berlin) und neun in Alkohol von Ambon (Bleeker).

62. Linckia pacifica Gray. Zwei Exemplare in Alkohol von Ambon (Bleeker).

63. Linckia miliaris (Linck) v. Martens. Acht getrocknete Exemplare von den Molukken, drei getrocknete ohne genauere Angabe von Herkunft als: Ost Indien, ein getrocknetes von Cates Bank (Frank), zehn in Alkohol von den Molukken (Bleeker), zwei in Alkohol von Bawean (Bleeker) und sechs in Alkohol von der Bai von Batavia (Sluiter).

64. Linckia guildingi Gray. Ein getrocknetes Exemplar von West-Indien (Eyken Sluyter) und zwei in Alkohol von den Antillen.

65. Nardoa novae caledoniae Perrier. Ein Exemplar in Alkohol von den Molukken. (Bleeker). Die eigentümliche Form der Armen, welche Perrier beschrieb ist an zwei Armen sehr deutlich zu unterscheiden, an den drei anderen aber nur sehr undeutlich. 
66. Nardoa pauciforis (v. Martens) Sladen. Ein Exemplar in Alkohol von Ambon (Bleeker). Das Tier stimmt in Hauptsache mit der Beschreibung von v. Martens überein, nur sind die Armen nicht platt, sondern gleich breit als hoch. Auf den Porenfeldern können noch bis 9 Poren vorkommen, gewöhnlich aber nicht mehr als 6. Es kommen zwei Madreporenplatten vor, eine grössere und eine kleinere in den beiden Armwinkeln desselben Armes. Die Rücken- Bauch- und Randplatten stimmen mit der Beschreibung von v. Martens gut überein. In einem der Armwinkel fand ich eine kleine Öffnung, und bei genauer Untersuchung stellte sich heraus, dass sich hier die eigentümliche von den Herrn Sarasin beschriebene parasitische Schnecke Stilifer linckiae Sar. eingenistet hatte.

67. Nardoa variolata (Retz.) Gray. Zwei Exemplare getrocknet von Neu-Ierland (Cumming) und ein Exemplar in Alkohol von Mauritius (Robillard).

68. Nardoa tuberculata Gray. Mehrere Exemplare in Alkohol von Ambon (Bleeker) und von der Bai von Batavia (Sluiter) und fünf getrocknet von den Molukken.

69. Nardoa semiregularis M. u. Tr. Zwei getrocknete Exemplare von den Molukken (v. d. Hucht).

\section{STICHASTERIDAE.}

70. Stichaster aurantiacus (Meyen) Verrill. Drei getrocknete Exemplare von Chili, und ein von Japan. Bis jetzt war diese Art nur noch von den Küsten von Chili und Peru bekannt. Das Exemplar von Japan stimmt aber so genau mit denen von Chili überein, dass ich es nicht von diesen zu unterscheiden wusste.

71. Stichaster roseus (O. F. Müller) Sars. Zwei Exemplare in Alkohol von Bergen (Weber).

\section{SOLASTERIDAE.}

72. Crossaster affinis (Brdt.) Sladen. Sehr zahlreiche Exemplare in Alkohol von verschiedenen Stellen der Barents See zwischen $70^{\circ}-76^{\circ} \mathrm{NB}$. und $19^{\circ}-44^{\circ}$ OL. Gr., aus einer Tiefe von 45-140 Faden. Unter den mehr als fünfaig Exemplaren war ein mit neun Armen und.ein mit elf. Wenn also auch die bei weitem gewöhnlichste Zahl der Arme zehn ist, so ist sie es doch nicht sinvariably", wie Koren und Danielssen angeben. Andrerseits steht es jetzt wohl ausser Zweifel, dass die genannten Autoren vollkommen recht haben, wenn sie C. affinis und C. papposus als zwei scharf gesonderten Arten aufführen. Nach dem Barents Material zu urteilen ist sogar der C. affinis in der Barents See weit häufiger als der C. papposus.

73. Crossaster papposus M. u. Tr. Zwei getrocknete Exemplare von der Küste von England (Damon) und zwei in Alkohol von der Barents See $70^{\circ} 17^{\prime}$ NB. und $46^{\circ} 31^{\prime}$ OL., Tiefe 45 Faden, und drei von Bodö, Norwegen'(Weber).

74. Solaster glacialis Dan. u. Kor. Ein Exemplar in Alkohol von der Barents See, $72^{\circ} 36^{\prime}$ NB. und $24^{\circ} 54^{\prime}$ OL., Tiefe 140 Faden.

75. Solaster endeca (Retz.) Forbes. Ein getrocknetes Exemplar von der Küste von Norwegen, neun Exemplare in Alkohol von der Barents See, zwischen $72^{\circ}-75^{\circ}$ NB. und $29^{\circ}-48^{\circ}$ OL., Tiefe 90-140 Faden, und ein Exemplar in Alkohol aus der Kara See (Varna Expedition) $71^{\circ}$ NB., 64. OL., und drei von Bodö, Norwegen (Weber).

76. Solaster intermedius (n. sp.). Ein Exemplar in Alkohol von der Barents See, 
$75^{\circ} 43^{\prime}$ NB. und $19^{\circ} 0^{\prime}$ OL. Gr. Tiefe 45 Faden. Boden: Steinen und Muschelschalen. Neun Arme, $\mathrm{R}=82 \mathrm{~mm}$., $\mathrm{r}=29 \mathrm{~mm}$. Die Scheibe ist mehr oder weniger gewölbt, die Arme platter, aber mit einer Andeutung eines medianen Kammes. Arme mässig lang, sich allmählig verjüngend, am Ende spitz und nach dem Rücken zu umgebogen. Die Armwinkel sind eckig, nicht ausgerundet. Bei jedem Armwinkel nimmt auf der Scheibe eine Furche (Sulcus) ihren Ursprung, welche zwar nicht scharf markiert, aber doch bis auf etwa die Hälfte der Scheibe deutlich $\mathrm{zu}$ verfolgen ist.

Der Rücken ist mit kurzen, breiten und stumpfen Paxillen besetzt, welche auf den Endflächen kleine Dörnchen tragen. Auf den Armen stehen die Paxillen, wenigstens auf den Seiten derselben, in regelmässigen schiefe Querreihen. In der Mitte der Arme, so wie auf der Scheibe stehen sie aber ganz regellos. Die Bases der Paxillen sind deutlich von einer dünnen Haut umgeben. Zwischen den Paxillen des Rückens sind zahlreiche Papulae zerstreut, welche nicht in Gruppen stehen.

Am Rande der Arme liegen zwei deutliche Reihen von Randplatten. Die ventralen liegen, wenigstens bei der Basis der Arme ganz auf der Bauchseite. Sie gleichen sehr grosse Paxillen, welche in der Richtung des Armradius stark zusammengedrückt sind. Die Dörnchen auf den Endflächen sind etwas grösser als die der Rückenpaxillen und auf jeder Platte kommen etwa 20 vor. Vom Armwinkel bis zur Spitze des Armes zählt man ungefähr 60 dieser ventralen Randplatten.

Die dorsalen Randplatten oder Randpaxillen sind zwar kleiner als die ventralen, aber doch deutlich zu unterscheiden und jedenfalls viel grösser als die Paxillen des Rückens. Sie wechseln ziemlich regelmässig mit den ventralen ab, sind aber etwas weniger in Anzahl, da nur 56 vorkommen.

Die Ambulacralplatten tragen eine sehr eigentümliche Bewaffnung. Auf jeder Platte steht eine Querreihe von vier, zuweilen fünf kräftigen Papillen, von welchen die der Fürche am nächsten stehende gewöhnlich die grösste ist. Die zweite, senkrecht zur ersten stehende Reihe von Papillen, welche der Ambulacralfurche parallel verläuft, und sonst immer bei Solaster vorkommt, ist aber rudimentär geworden. Nur liegt ziemlich tief in der Ambulacralfurche versteckt auf jeder Platte eine mässig lange Papille, zu welcher sich zuweilen noch eine zweite kleinere hinzugesellt. Es scheint, dass diese die letzten Überbleibsel sind der zweiten für Solaster sonst so typischen Papillenreihe.'Alle diese Papillen sind mit einer ziemlich dicken Haut überzogen.

Die Mundplatten sind gross. Die Bewaffnung besteht aus einer Randreihe von 12 Papillen, von welchen die dem Munde am nächsten liegenden die grössten sind. In der Mitte jeder Platte kommt' noch eine Gruppe von acht Papillen hinzu, welche nur undeutlich zwei Reihen bilden, und von welchen auch die dem Munde am nächsten liegenden die grössten sind, obgleich alle kleiner bleiben als die Randpapillen. Auch alle diese Papillen sind von einer dicken Haut überzogen.

Die ventralen Interambulacralplatten bilden ziemlich breite dreieckige Felder, sind dicht mit Paxillen besetzt, welche beträchtlich grösser sind als die des Rückens, und auf den Endflächen fünf bis acht Dörnchen tragen. Auch an der Basis der Arme schieben sie sich bis auf etwa $1 / 3$ der Armlänge zwischen den Ambulacralplatten und ventralen Randplatten ein.

Die Madreporenplatte ist sehr klein, fast gänzlich unter den Paxillen verborgen, und dem Rande etwas näher liegend als der Mitte der Scheibe.

Das Skelett des Rückens besteht aus sternförmigen Platten, welche durch schmale Verbindungsstücken mit einander zusammenhängen und ziemlich kleine unregelmässig drei- oder viereckige Felder zwischen sich frei lassen.

Die Ambulacralfüsschen stehen in zwei Reihen und haben gut entwickelte fleischige Endscheiben.

Diese Art nimmt eine gewisse Zwischenstellung ein, zwischen den verschiedenen Genera, welche von verschiedenen Autoren in der Familie der Solasteridae unterschieden werden und 
zuletzt von Sladen scharf umschrieben sind. So gleicht die Bewaffnung der Ambulacralplatten derjenigen von Korethraster und Peribolaster. Andrerseits hat unsere Form die beiden wohl entwickelten Reihen von Randpaxillen gemein mit der Gattung Lophaster, die Structur des dorsalen Skeletts und die dicht stehenden Paxillen des Rückens hingegen wieder mit Solas.ter, indem die zahlreichen Papulae auf dem Rücken wieder an Crossaster erinnern, wie diese verschiedenen Genera jetzt von Slad en characterisiert sind.

Ich war desshalb in nicht geringer Verlegenheit, als ich diese neue Form unter einer dieser Genera unterbringen wollte. Andrerseits scheint mir aber der Wert von Gattungsmerkmäler ein sehr zweifelhafter, wenn wir die Kennzeichen, durch welche die verschiedenen Genera von einander unterschieden werden sollen, bei einer und derselben Form vereinigt finden, wie es bei Solaster intermedius der Fall ist. Wenn nun noch dazu verschiedene Autoren, welche anerkannte Autoritäten auf diesem speciellen Gebiet sind, zu ganz entgegengesetzten Resultaten kommen, so dass Danielssen und Koren alle Formen in einem einzigen Genus vereinigen, indem Perrier und Sladen mehrere Gattungen anerkennen, so scheint es mir für den Augenblick am besten, diese neue Form zu der alten Gattung Solaster zu bringen.

77. Lophaster furcifer (Düb. und Kor.) Verrill. Vier Exemplare aus der Barents See in Alkohol, $75^{\circ} 20^{\prime}$ NB. und $46^{\circ} 40^{\prime}$ OL. Tiefe 150 Faden, und zehn Exemplare in Alkohol aus der Kara See (Varna Expedition) $71^{\circ} \mathrm{NB}$. und $64^{\circ} \mathrm{OL}$.

\section{PTERASTERIDAE.}

78. Pteraster militaris M. u. Tr. Zahlreiche Exemplare in Alkohol aus der Barents See, $72^{\circ}-77^{\circ}$ NB. und $15^{\circ}-48^{\circ}$ OL., Tiefe $45-180$ Faden, und ein von Bodö, Norwegen (Weber).

79. Pteraster pulvillus Sars. Zwei Exemplare in Alkohol aus der Barents See, $72^{\circ} 34^{\prime}$ NB. und $48^{\circ} 6^{\prime}$ OL., Tiefe 140 Faden, und ein in Alkohol aus der Kara See $71^{\circ} 19^{\prime}$ NB. und $63^{\circ} 56^{\prime}$ OL., Tiefe 56 Faden (Varna Expedition).

80. Hymenaster pellucidus W. Thomp. Sechs Exemplare in Alkohol aus der Kara See $71^{\circ} 19^{\prime}$ NB. und $63^{\circ} 56^{\prime}$ OL., Tiefe 56 Faden (Varna Expedition).

\section{ECHINASTERIDAE.}

81. Acanthaster echinites Lütk. Mehrere Exemplare von den Molukken in Alkohol (v. d. Hucht, Bleeker). Unter diesen ein sehr junges Tier.

82. Mithrodia clavigera (Lam.) Perrier. Drei getrocknete Exemplare von den Molukken (v. d. Hucht) und ein ebenso getrocknetes von Neu Ierland.

83. Cribrella oculata (Linck) Forbes. Zahlreiche Exemplare in Alkohol von sehr verschiedenen Stellen der Barents See, aus Tiefen von 84-155 Faden, zwei in Alkohol von Roscoff (Kerbert), viele von Bodö (Weber), zwei getrocknete von der Küste von England (Damon), und drei getrocknete von der Küste von Grönland (Israel).

84. Echinaster spinosus (Retz.) M. u. Tr. Zwei Exemplare, getrocknet von Japan (Kock Blomhoff), und ein in Alkohol von der Küste von Florida (Agassiz, Reichs Museum). Dem Etiquette nach sollen die zwei erst erwähnten Tiere von Japan herstammen, was allerdings sehr merkwürdig sein würde, da bis jetzt der E. s pinosus nur noch von der Ost Küste Amerikas, von Virginia, Yucatan, Bahia und Rio Janeiro bekannt ist. Die Tiere stimmen genau mit dem von Agassiz bei Florida gesammelten Exemplar überein nnd ich habe für den Augenblick keinen Grund an die Richtigkeit der Etiquette zu zweifeln. 
85. Echinaster purpureus (Gray) Bell. Vier Exemplare, getrocknet von den Molukken (v. d. Hucht), and vier Exemplare in Alkohol von Mauritius (Robillard).

86. Echinaster eridanella M. u. Tr. Mehrere Exemplare in Alkohol von den Molukken (Bleeker).

87. Echinaster sepositus M. und Tr. Ein Exemplar von Roscoff. (Kerbert), ein von Rovigno und ein von unbekannter Herkunft, alle in Alkohol.

\section{HELIASTERIDAE.}

88. Heliaster helianthus (Lam.) Duj. et Hupé. Vier getrocknete Exemplare von Chili.

\section{ASTERIDAE.}

89. Asterias mülleri Sars. Vier Exemplare in Alkohol aus der Nord See vom Doggersbank.

90. Asterias gelatinosa Meyen. Zwei getrocknete Exemplare von Valparaiso.

91. Asterias polaris M. u. Tr. Ein getrocknetes Exemplar von Grönland (Conradsen).

92. Asterias rubens L. Mehrere Exemplare aus der Nord See, zum Teil getrocknet, zum Teil in Alkohol, und mehrere Exemplare von Hammerfest in Alkohol (Weber).

93: Asterias glacialis O. F. Müll. Ein getrocknetes Exemplar von Grönland, vier getrocknete Exemplare von Madeira (Frank), und ein in Alkohol von Roscoff (Kerbert).

94. Asterias linckii M. und Tr. Zahlreiche Exemplare in Alkohol von verschiedenen Stellen der Barents See, aus Tiefen von 80-150 Faden.

95. Asterias stellionura Perrier. Ein Exemplar aus der Barents See, $71^{\circ} 8^{\prime}$ NB. und $44^{\circ} 25^{\prime}$ OL., Tiefe 80 Faden.

96. Asterias gunneri Dan. u. Kor. Ein Exemplar in Alkohol aus der Barents See, $71^{\circ} 8^{\prime}$ NB. und $44^{\circ} 25^{\prime}$ OL., Tiefe 80 Faden.

97. Asterias panopla Stuxb. Zahlreiche Exemplare in Alkohol aus dem Nördlichen Teil der Barents See, $77^{\circ} 7^{\prime}$ NB. und $49^{\circ} 37^{\prime}$ OL. und von anderen nahe liegenden Localitäten. Gleichfalls mehrere Exemplare aus der Kara See (Varna Expedition).

98. Asterias groenlandica (Lütk.) Stimps. Zahlreiche Exemplare in Alkohol von verschiedenen aber mehr südlichen Stellen der Barents See, $69^{\circ}-70^{\circ} \mathrm{NB}$, und $19^{\circ}-54^{\circ}$ OL., Tiefe 55-62 Faden.

99. Asterias gemmifera Perrier. Zwei getrocknete Exemplare von Madura (Frank).

100. Asterias tenera Stimpson. Ein Exemplar in Alkohol aus der Massachussett Bai (Agassiz, Reichs Museum).

101. Asterias forbesi (Desor) Verrill. Zwei Exemplare aus der Nord Atlantischen Ocean und ein von Charleston (Agassiz, Reichs Museum), alle in Alkohol.

A msterdam, Januar 1894. 